\title{
Diversidad de moluscos gasterópodos terrestres en la región del Pacífico de Nicaragua y sus preferencias de hábitat
}

\author{
Antonio Mijail Pérez ${ }^{1}$, Marlon Sotelo ${ }^{1}$, Irma Arana ${ }^{1}$ \& Adolfo López ${ }^{2}$ \\ Asociación Gaia, Managua, Nicaragua; mijail@ibw.com.ni. Telefax: 505-249 3127. \\ Universidad Centroamericana, Centro de Malacología y Diversidad Animal (CEMADIA), Managua, Nicaragua. \\ Recibido 21-II-2006. Corregido 27-I-2007. Aceptado 25-VII-2007.
}

\begin{abstract}
Diversity of terrestrial gastropod mollusks in the Pacific region of Nicaragua, and their habitat preferences. Landsnail communities of the Nicaraguan Pacific Slope were studied. The study area was subdivided into 21 quadrats (ca. 40 x $40 \mathrm{~km}$ ). A high taxonomic richness was found: 79 species, 43 genera and 23 families. Species richness (S) per quadrat ranged from 17 through 50. The biogeographic index presented higher values on quadrats 5 (3.04), $6(3.03), 8(2.96)$ and 11(2.96). Quadrat 11 had the highest species richness $(\mathrm{S}=50)$, and one of the highest biogeographic index values ( $\mathrm{IB}=2.96)$. Gastropod mollusk communities are favored by sites with an illumination of filtered sun, associated to riparian forests and with a susbtratum of wet soils and leaf litter. Rev. Biol. Trop. 56 (1): 317-332. Epub 2008 March 31.
\end{abstract}

Key words: terrestrial gasteropods, Nicaragua, diversity, habitat.

Según Pérez et al. (1996), los estudios comunitarios o a nivel de paisajes en moluscos $\mathrm{u}$ otros invertebrados terrestres en general son escasos. Como señala Austin (1985), estos se han centrado a nivel global esencialmente en comunidades de vertebrados terrestres. Sin embargo, existen trabajos como los de Cameron (1978, 1982, 1986), Walden (1981), y Cowie et al. (1995) sobre comunidades de moluscos terrestres que tienen un gran valor metodológico. Así mismo, se debe mencionar la contribución de Getz y Uetz (1994), quienes realizaron un estudio similar al presente en la zona sur de las montañas Apalaches. La fauna de moluscos continentales de la región del Pacífico de Nicaragua ha sido relativamente bien estudiada desde el punto de vista taxonómico (Pérez 1999, Pérez y López 1999, 2002). Estos autores han realizado un inventario detallado de los gasterópodos continentales de esta región del país utilizando el método cartográfico UTM, así como una caracterización taxonómica completa de algunas de las especies más relevantes de la zona. Existen sin embargo, escasos estudios cuantitativos de la diversidad en el nivel de comunidades, así como caracterizaciones del hábitat de las especies, y los existentes abordan mayormente el estudio de fauna de vertebrados (vid. Medina et al. 2004, Salgado y Páiz 2004, Vílchez et al. 2004).

A pesar de la escasez de este tipo de estudios, los mismos constituyen la base para la valoración del potencial de conservación de áreas geográficas de interés y el establecimiento de prioridades de conservación, porque en estas valoraciones se deben considerar al unísono los criterios de diversidad de las comunidades y el valor biogeográfico o endemismo de las especies que las componen (Pérez y López 1995).

En el presente trabajo se estudiaron las comunidades de moluscos gasterópodos continentales de la región del Pacífico de Nicaragua. Para la realización de este trabajo se utilizó el método de cartografiado UTM mediante el cual el área de estudio se dividió en 21 cuadrículas de aproximadamente la misma área 
(ca. 40x40 km), estas cuadrículas se obtuvieron por la fusión de las cuadrículas de 10x10 km en las que se tomaron los datos de campo. Para los detalles del método ver el trabajo de Pérez y López (2003). A las comunidades de cada cuadrícula se les determinó su composición taxonómica y se les calcularon algunos índices para su caracterización ecológica y biogeográfica. Además se estudiaron diferentes variables del hábitat para determinar cuál es el mejor escenario para el desarrollo de comunidades de gasterópodos terrestres.

\section{MATERIALES Y MÉTODOS}

Área de estudio: Nicaragua con una superficie terrestre de $118358 \mathrm{~km}^{2}$, se encuentra dividida en tres zonas geográficas principales: 1. La zona del Pacífico $\left(38700 \mathrm{~km}^{2}\right)$, 2. El triangulo montañoso de las tierras altas del interior o región central-norte (42 $\left.400 \mathrm{~km}^{2}\right)$, 3. La planicie costera del Atlántico, que consiste en una ancha faja de planicies onduladas que bordean la costa Atlántica (46 $600 \mathrm{~km}^{2}$ ). Además, Nicaragua tiene dos grandes lagos: el lago de Managua $\left(1040 \mathrm{~km}^{2}\right)$ y el lago de Nicaragua (8 $200 \mathrm{~km}^{2}$ ), que constituyen el $7.6 \%$ del territorio nacional. Dentro de este contexto, la región del Pacífico (Fig. 1) tiene un área aproximada de $38700 \mathrm{~km}^{2}$, pero descontando el área ocupada por los lagos queda en $29460 \mathrm{~km}^{2}$.

Muestreos: para la recolección de los datos se utilizó el método cartográfico UTM como se detalla en el trabajo de Pérez y López (2003). Se recorrió el territorio escogiendo puntos o estaciones de recolecta arbitrariamente dentro de cuadrículas UTM de 10x10 km; de tal forma que se seleccionaron aquellos con características adecuadas para el desarrollo de una comunidad de gasterópodos. Las recolectas se realizaron siempre por debajo de los 500 msnm ya que la región del Pacífico es mayoritariamente llana. Según Pérez (1999), los gasterópodos tienen preferencia por ambientes relativamente húmedos, sombríos y con un alto contenido de materia orgánica. De modo que para los puntos de recolecta se prefirieron sitios que contenían al menos un árbol, con hojarasca o vegetación herbácea, presencia de rocas y troncos caídos; procurando seleccionar de esta forma hábitat idóneos para obtener un máximo de muestras.

Se realizaron de dos a tres estaciones de recolecta dentro de cada cuadrícula de 10x10 $\mathrm{km}$, como recomiendan Marquet (1985) y Altonaga et al. (1994). El periodo de recolecta para una estación fue de una hora y el número de recolectores fue de cuatro personas. El número de recolectores (4) ha sido concebido para compensar la deficiencia que podría presentarse al extrapolar sin modificaciones un método hasta el presente usado por autores europeos al estudio de comunidades faunísticas neotropicales. De tal suerte, teniendo cuatro recolectores se triplica o cuadriplica el esfuerzo de muestreo propuesto por Marquet (1985), quien se refiere a un recolector.

El muestreo se llevó a cabo durante la estación lluviosa, porque se ha comprobado que en

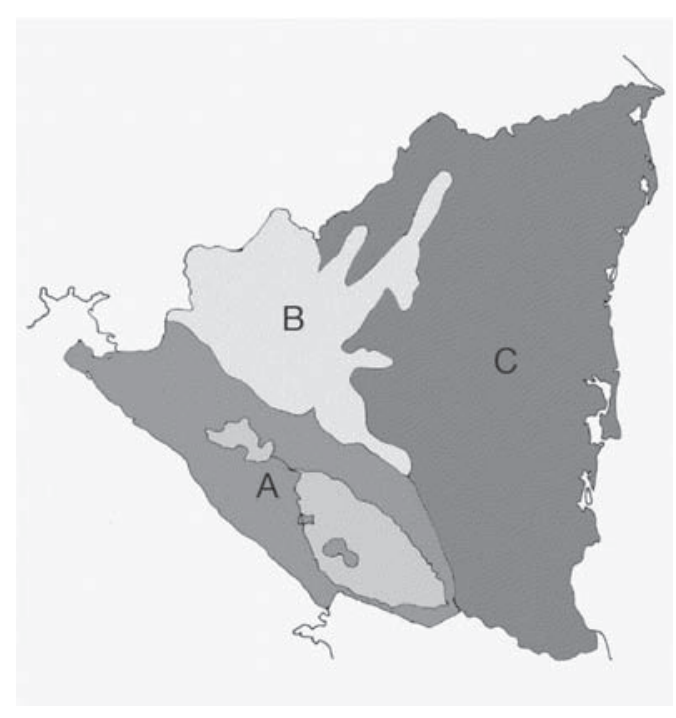

Fig. 1. A: El Pacífico de Nicaragua según Oviedo (1993) e Incer (1973); B: Región Centro Norte, C: Región Atlántica.

Fig. 1. A: The Nicaraguan Pacific slope, according to Oviedo (1993) and Incer (1973); B: Central-North region, C: Atlantic region. 
las mismas comunidades locales de gasterópodos, en la región del Pacífico de Nicaragua, existen variaciones estacionales entre ambas estaciones del año (lluvia y seca); sin embargo, la comunidad de lluvia contiene por completo a la comunidad de seca y tiene además una mayor riqueza de especies (Pérez, obs. pers.).

De cara al análisis de los datos, el área de estudio (Pacífico de Nicaragua) se dividió nuevamente en 72 cuadrículas de 20×20 km que resultan de la fusión de cuatro de las cuadrículas de 10x10 km las cuales se utilizaron originalmente para la recolección de los datos de campo. Para realizar esta fusión se tomaron las cuatro cuadrículas más próximas partiendo del extremo occidental del área de estudio y eligiendo siempre de oeste a este y de norte a sur. No obstante, debido a la gran cantidad de cuadrículas y para darle mayor coherencia al análisis de los resultados se redujo el número de cuadrículas reagrupando nuevamente las cuadrículas de 20x20 km más próximas siguiendo el criterio antes empleado e intentando formar nuevas cuadrículas de tamaño similar. Este sistema nos permite además aumentar la cantidad de especies contenidas en cada cuadrícula. Finalmente el área de estudio quedo dividida en 21 cuadrículas (Fig. 2) de aproximadamente 40x40 km. Los datos del hábitat se tomaron usando el protocolo de Marquet (1985), simplificado.

Se recolectó material seco (conchas vacías) y material vivo. Los especímenes vivos fueron relajados en mentol y fijados en alcohol de $70 \%$, como sugiere Calvo (1994). Ambos tipos de materiales se incluyeron en la colección del Centro de Malacología y Diversidad Animal de la Universidad Centroamericana. La identificación de las especies se realizó con base en la obra de Pérez y López (2002) y las Colecciones Malacológicas depositadas en el Centro de Malacología y Diversidad Animal de la Universidad Centroamericana.

Hábitat: para la identificación de las formaciones vegetales se siguieron los criterios de Salas (1993, simplificado) Las formaciones

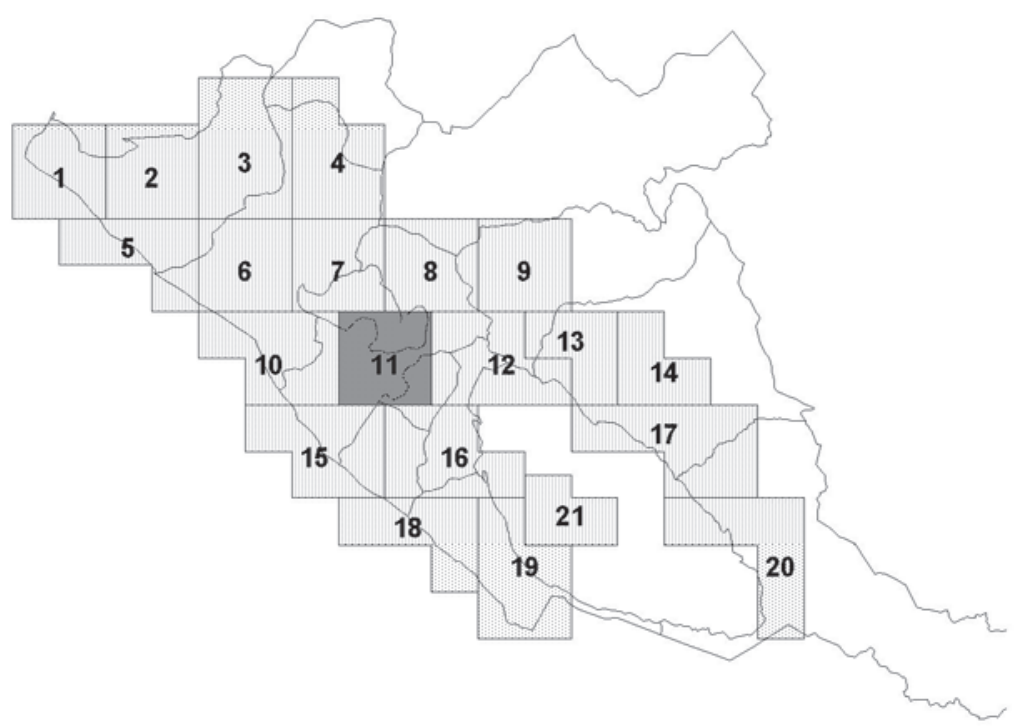

Fig. 2. Área de estudio dividida en 21 cuadrículas. La "cuadrícula 21" es la isla de Ometepe, Dpto de Rivas, que se localiza dentro del lago de Nicaragua, contigua a la cuadrícula 19 y queda fuera de la trama cuadriculada. Cuadrícula 11 con el valor más alto de riqueza de especies $(\mathrm{S}=50)$.

Fig. 2. Study area divided into 21 quadrats. "Quadrat 21", is Ometepe island, Rivas Dept., located in Lake Nicaragua, besides quadrat 19 and outside the numbered frame. Quadrat 11 with the highest species richness value $(\mathrm{S}=50)$. 
vegetales visitadas fueron las siguientes: Bosques bajos o medianos sub-caducifolios (BBMCS); Bosques bajos o medianos caducifolios (BBMC); Bosques medianos o altos perennifolios (BMAP); Bosques medianos o altos sub-perennifolios (BMASP); Bosques bajos de esteros y marismas (BBEM); Bosque bajo con matorral abundante (BBMA); Bosques bajos de sitios inundados periódicamente (BBSIP); Bosques de galería (BG); Sabanas (S); Sabanas de Crescentia alata (JI); Arboledas (ARB); Tipos de asentamientos humanos: Cercas vivas (CV) (Según Murgueitio et al. (2003) estas son filas de árboles creciendo libremente en múltiples estratos, con al menos un estrato superior de $4 \mathrm{~m}$ de alto y $4 \mathrm{~m}$ de copa; Patios (Pat), Cauces (Cau), Puentes (Pts), Terrenos de pastoreo (Past) y Plantaciones (Plants); Tipo de suelo (Con hojarasca, Sin hojarasca, Con humus, Sin humus, Arena, Hojarasca con arcilla, Hojarasca con arena y Grava volcánica); Humedad del suelo (Saturado, Húmedo suelto, Húmedo compacto, Seco suelto y Seco compacto), así como aspectos climáticos como la iluminación (Sol abierto, Parches de sol, Sol filtrado, Penumbra y Umbra).

Análisis numérico: se calculó el índice (S) de riqueza de especies (Ludwig y Reynolds 1988) que no es más que la cantidad de especies presentes en este caso en una cuadrícula de 40x40 km; se cuantificaron los individuos totales, vivos y/o conchas. Lo que en este caso sería una medida de la diversidad gamma de la región estudiada de acuerdo a Brown y Lomolino (1998) y Magurran (1987). Se estimó también la abundancia de individuos vivos en cada cuadrícula.

Se calculó además el índice biogeográfico IB (Pérez et al. 1996), este cuantifica la calidad de las especies que componen la comunidad. Para esto las especies encontradas fueron agrupadas en las siguientes categorías biogeográficas: especies endémicas (5), especies centroamericanas (4), especies antillanas (3), especies norteamericanas y suramericanas (2) y especies de amplia distribución (1).

A los datos obtenidos tanto de frecuencia por formaciones vegetales como de frecuencia por variables del hábitat, se les aplicó la prueba de bondad de ajuste $\mathrm{x}^{2}$ para cada una de las variables.

\section{RESULTADOS}

Composición: se recolectaron un total de 79 especies distribuidas en 43 géneros y 23 familias. Estos resultados indican que esta región contiene una alta riqueza de especies, géneros y familias (Anexo 1).

En general la riqueza de especies observada es alta, correspondiendo los valores más altos a la cuadrícula número $11(\mathrm{~S}=50)$ (Fig. 2) y los valores más bajos corresponden a la cuadrícula $1(\mathrm{~S}=17)$. En este sentido, Solem y Climo (1985) plantearon que las comunidades de moluscos están normalmente compuestas por cantidades entre cinco y 12 especies, aunque en este caso se trata de una escala territorial más amplia o escala de paisaje.

Del total de las especies estudiadas 12 son especies endémicas (15.19\%), 21 especies centroamericanas $(26.58 \%)$, siete especies antillanas $(8.90 \%), 24$ especies norteamericanas y suramericanas $(30.38 \%)$ y 15 especies de amplia distribución (19\%). El índice biogeográfico presentó los valores más altos en las cuadrículas 5 (3.04), 6 (3.03), 8 (2.96) y 11 (2.96) (Cuadro 1). Estos valores indican que en estas comunidades se encuentran las especies con mayor valor de endemismo y por tanto son las comunidades con mayor valor biogeográfico, lo cual es un dato a considerar de cara a la conservación de estos sitios.

Abundancia: los valores de abundancia total de las especies en las cuadrículas muestreadas se presentan en el Cuadro 2. Como se puede observar las especies dominantes en el área de estudio son, en ese orden: Subulina octona (1021), Bulimulus corneus (931), Beckianum beckianum (906), Lamellaxis gracilis (893), Praticolella griseola (828) y Lamellaxis micra (494).

Hábitat: al analizar el tipo de vegetación (Fig. 3), encontramos que existe una 
CUADRO 1

Índices ecológicos calculados en las comunidades de moluscos gasterópodos terrestres del Pacifico de Nicaragua

TABLE 1

Ecological indexes calculated on terrestrial gastropod mollusks communities from the Nicaraguan Pacific slope

\begin{tabular}{|c|c|c|c|}
\hline Cuadricula & $\begin{array}{c}\text { Índice } \\
\text { biogeográfico }\end{array}$ & $\begin{array}{c}\text { Riqueza } \\
\text { total }\end{array}$ & $\begin{array}{c}\text { Riqueza } \\
\text { vivos }\end{array}$ \\
\hline 1 & 2.7647 & 17 & 8 \\
\hline 2 & 2.75 & 24 & 15 \\
\hline 3 & 2.921 & 38 & 26 \\
\hline 4 & 1.4657 & 38 & 20 \\
\hline 5 & 3.0416 & 24 & 12 \\
\hline 6 & 3.0322 & 31 & 13 \\
\hline 7 & 2.8181 & 22 & 13 \\
\hline 8 & 2.9629 & 27 & 4 \\
\hline 9 & 2.9333 & 45 & 12 \\
\hline 10 & 2.8076 & 26 & 15 \\
\hline 11 & 2.96 & 50 & 19 \\
\hline 12 & 2.7105 & 38 & 15 \\
\hline 13 & 2.6976 & 43 & 17 \\
\hline 14 & 2.6578 & 38 & 11 \\
\hline 15 & 2.923 & 26 & 16 \\
\hline 16 & 2.9387 & 49 & 20 \\
\hline 17 & 2.6944 & 36 & 11 \\
\hline 18 & 2.5 & 24 & 10 \\
\hline 19 & 2.6857 & 35 & 14 \\
\hline 20 & 2.8257 & 29 & 10 \\
\hline 21 & 2.7173 & 46 & 17 \\
\hline $\mathrm{X}$ & 2.7527 & 33.619 & 14.19 \\
\hline DS & 0.33 & 9.58 & 4.80 \\
\hline
\end{tabular}

preferencia muy significativa por el Bosque de Galería $\left(\mathrm{x}^{2}=94.07, \mathrm{p}<0.01,10 \mathrm{gl}\right)$.

Asentamientos humanos: la abundancia por asentamientos humanos (Fig. 4), denota una preferencia muy significativa por las Cercas vivas $\left(\mathrm{x}^{2}=255.91, \mathrm{p}<0.01,5 \mathrm{gl}\right)$, lo cual es comprensible si se tiene en cuenta que la región del Pacífico de Nicaragua está profundamente antropizada y las Cercas vivas constituyen el tipo de uso de suelo más emblemático para la conservación de la biodiversidad en la región.

Tipo y humedad del suelo: respecto al tipo del suelo (Fig. 5), se observa una preferencia muy significativa por el suelo con hojarasca $\left(x^{2}=252.60, p<0.01,7 \mathrm{gl}\right)$. En relación con las condiciones de humedad los resultados expresan una preferencia muy significativa por el suelo húmedo suelto $\left(\mathrm{x}^{2}=36.94, \mathrm{p}<0.01,4 \mathrm{gl}\right)$ (Fig. 6).

Iluminación: la mayor parte de los individuos fueron encontrados en condiciones de Iluminación de sol filtrado $\left(\mathrm{x}^{2}=17.66, \mathrm{p}<0.01\right.$, 4 gl) (Fig. 7).

\section{DISCUSIÓN}

Composición de especies y diversidad: el valor promedio de riqueza de especies observada fue de aproximadamente 34 especies, con una riqueza mínima de 17 especies (Cuadrícula 1) y una riqueza máxima de 50 (Cuadrícula 11). Estos valores de riqueza de especies pueden ser considerados altos a pesar de que la malacofauna de Nicaragua fue considerada pobre por Fischer y Crosse (1870-1902) y por Martens (1890-1901), así como más recientemente por Jacobson (1968).

El valor más alto de riqueza de especies $(S=50)$ se ubica en una cuadrícula donde están contenidas algunas de las zonas más conservadas de la región del Pacífico de Nicaragua, como la Laguna de Apoyo y la Península de Chiltepe. Ambas se encuentran dentro del Sistema de Áreas Protegidas de Nicaragua bajo la categoría de Reservas Naturales (MARENA 1999).

No obstante, se debe tener en cuenta que la región del Pacífico se encuentra en condiciones de avanzada deforestación y en general de antropización ya que es donde existe la mayor densidad poblacional de todo el país, con unos 133.9 habitantes/ $\mathrm{km}^{2} \mathrm{y}$ una población total de 2467742 (INEC 1995). Posiblemente en 


\section{CUADRO 2}

Abundancias de las especies recolectadas en el área de estudio considerando sólo ejemplares vivos

TABLE 2

Abundances of species collected on the study area, considering only living specimens

\begin{tabular}{|c|c|c|c|c|c|}
\hline Especies & Abundancias & Especies & Abundancias & Especies & Abundancias \\
\hline $\operatorname{Hr}(4)$ & 48 & $\operatorname{Li}(3)$ & 22 & $\operatorname{Tco}(2)$ & 22 \\
\hline $\mathrm{Ht}$ & 21 & $\operatorname{Lint}(4)$ & 100 & $\operatorname{Pgr}(2)$ & 828 \\
\hline $\mathrm{Ll}(2)$ & 141 & $\operatorname{Lla}(3)$ & 26 & $\operatorname{Tca}(4)$ & 22 \\
\hline $\mathrm{Nd}(5)$ & 95 & $\operatorname{Lta}(2)$ & 21 & $\mathrm{~T} \cos (4)$ & 28 \\
\hline $\operatorname{Ssp}(5)$ & 21 & $\operatorname{Lsp}(5)$ & 21 & $\operatorname{Tcr}(2)$ & 69 \\
\hline $\mathrm{Bco}(2)$ & 21 & Opu(1) & 51 & Tho(2) & 69 \\
\hline $\operatorname{Bte}(2)$ & 21 & Pssp(5) & 21 & $\mathrm{Xta}(2)$ & 21 \\
\hline Pdi(1) & 21 & $\operatorname{Soc}(1)$ & 1021 & $\operatorname{Tpl}(1)$ & 21 \\
\hline $\operatorname{Pmi}(3)$ & 21 & $\operatorname{Hbi}(1)$ & 40 & $\mathrm{Bco}(4)$ & 931 \\
\hline Pmed (2) & 21 & $\operatorname{Ecu}(4)$ & 27 & $\operatorname{Dal}(4)$ & 21 \\
\hline $\operatorname{San}(1)$ & 25 & $\operatorname{Eob}(5)$ & 37 & $\operatorname{Ddi}(4)$ & 60 \\
\hline $\operatorname{Vmi}(2)$ & 21 & Pun(4) & 21 & Ddo(1) & 22 \\
\hline Gge(2) & 21 & $\operatorname{Sgu}(4)$ & 21 & $\operatorname{Dmu}(2)$ & 21 \\
\hline $\operatorname{Ggu}(5)$ & 80 & Shi(2) & 21 & $\operatorname{Dtr}(4)$ & 21 \\
\hline Gpel(3) & 23 & $\operatorname{Spe}(2)$ & 21 & Datt (2) & 21 \\
\hline Gpen(2) & 21 & Spsp(5) & 21 & $\mathrm{Ofe}(2)$ & 21 \\
\hline Gse(3) & 24 & $\operatorname{Dla}(1)$ & 28 & Opr(4) & 24 \\
\hline $\operatorname{Sgu}(4)$ & 21 & Epi(4) & 55 & Dpi(4) & 21 \\
\hline Sre(4) & 112 & Ggu(1) & 71 & Mop(5) & 21 \\
\hline $\operatorname{Cco}(4)$ & 117 & $\operatorname{Hch}(4)$ & 21 & $\mathrm{Mpa}(4)$ & 40 \\
\hline $\operatorname{Cgu}(3)$ & 22 & Hse(1) & 23 & $\mathrm{Pbu}(4)$ & 21 \\
\hline $\operatorname{Bbe}(1)$ & 906 & $\mathrm{Htr}(4)$ & 21 & Cpi(2) & 21 \\
\hline $\operatorname{Bsi}(5)$ & 217 & $\operatorname{Gin}(2)$ & 97 & Rmi(2) & 21 \\
\hline $\operatorname{Bsp}(5)$ & 21 & $\operatorname{Gsp}(5)$ & 88 & $\operatorname{Rsp}(5)$ & 21 \\
\hline $\operatorname{Lgr}(1)$ & 893 & $\operatorname{Hmi}(1)$ & 21 & $\operatorname{Doc}(3)$ & 70 \\
\hline $\operatorname{Lmi}(1)$ & 494 & Sme(2) & 21 & $\operatorname{Lfl}(1)$ & 21 \\
\hline $\operatorname{Lgu}(4)$ & 42 & & & & \\
\hline
\end{tabular}

*El número entre paréntesis se refiere al valor de endemismo de la especie en la escala utilizada, previamente explicada en los métodos. Las abreviaturas para las especies aparecen en el Anexo 1.

*The figure between parentheses refers to the value of endemism according to the scale and explained in the methods. Species abbreviations are presented on Annex 1. 


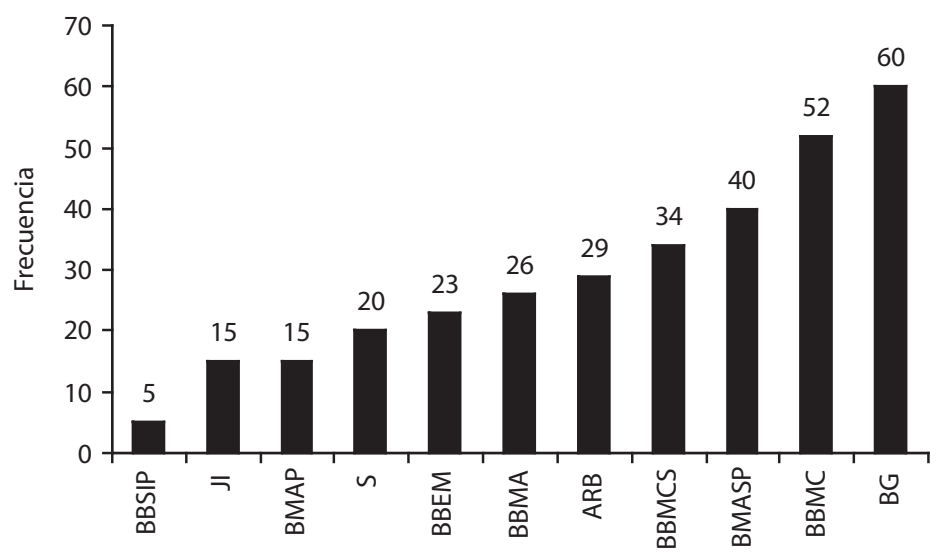

Tipos de vegetación

Fig. 3. Preferencia de las especies por las formaciones vegetales estudiadas. Bosques bajos o medianos subcaducifolios (BBMCS); Bosques bajos o medianos caducifolios (BBMC); Bosques medianos o altos perennifolios (BMAP); Bosques medianos o altos subperennifolios (BMASP); Bosques bajos de esteros y marismas (BBEM); Bosque bajo con matorral abundante (BBMA); Bosques bajos de sitios inundados periódicamente (BBSIP); Bosque de galería (BG); Sabana (S); Sabana de Crescentia alata (JI); Arboledas (ARB).

Fig. 3. Preference of species for the studied plant communities. Low to median semi-deciduous forests (BBMCS); Low to median deciduous forests (BBMC); Median to high perennial forests (BMAP); Median to high sub-perennial forests (BMASP); Low forests from marshes (BBEM); Low forests with shrubs (BBMA); Low forests from periodically flooded sites (BBSIP); Riparian Forests (BG); Savannah (S); Savannah of Crescentia (JI); Woodland (ARB).

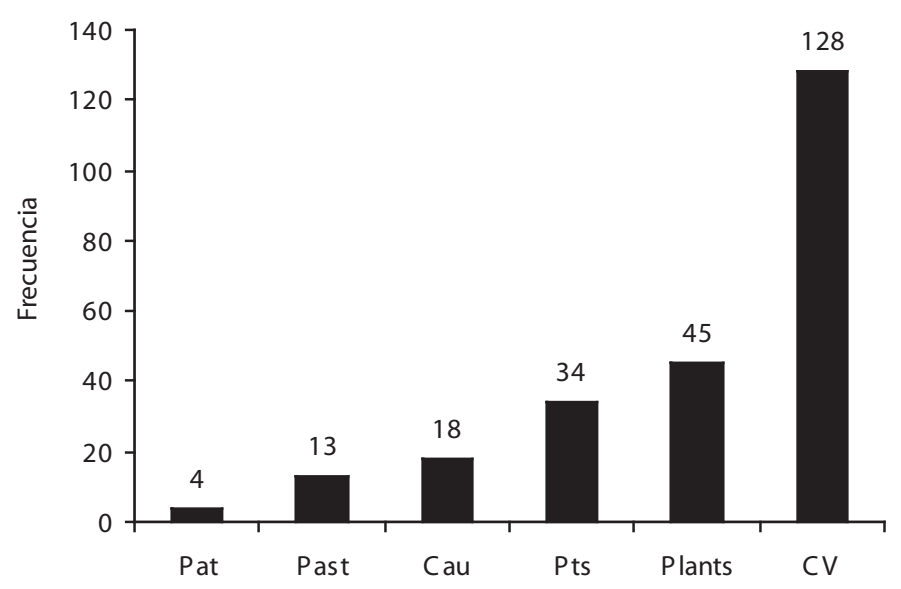

Asentamientos humanos

Fig. 4. Preferencia de las especies por los asentamientos humanos estudiados. Cercas vivas (CV); Patios (Pat), Cauces (Cau), Puentes (Pts), Terrenos de pastoreo (Past) y Plantaciones (Plants).

Fig. 4. Preference of species for the studied human settlements. Live fences (CV); Backyards (Pat), Ditches (Cau), Bridges (Pts), Grasslands (Past) y Plantations (Plants). 


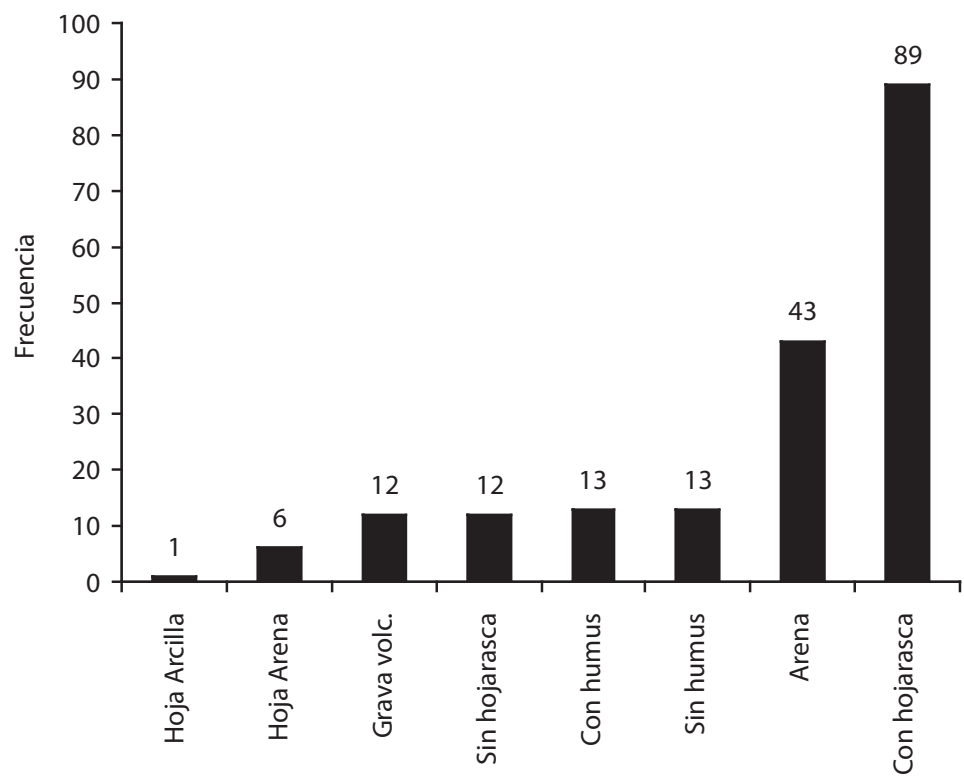

Tipos de suelos

Fig. 5. Preferencia de las especies por el tipo de suelo. Con hojarasca, Sin hojarasca, Con humus, Sin humus, Arena, Hojarasca con arcilla, Hojarasca con arena y Grava volcánica.

Fig. 5. Preference of species for the soil type. With litter, No litter, With debris, No debris, Sand, Clay with litter, Sand with litter and Volcanic gravel.

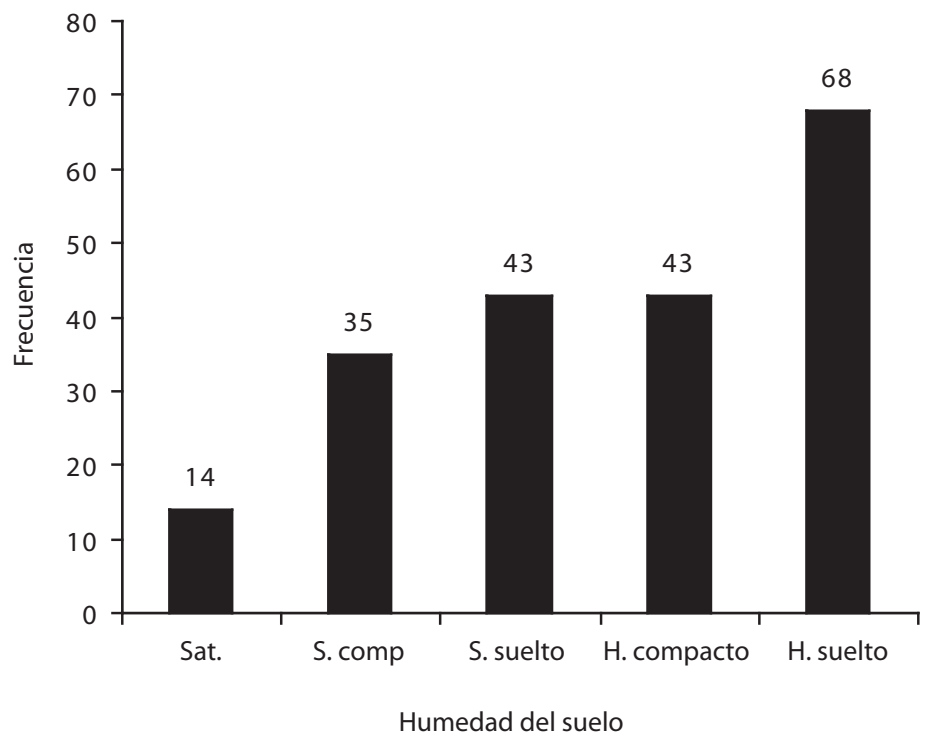

Fig. 6. Preferencia de las especies por la humedad del suelo. Saturado, Húmedo suelto, Húmedo compacto, Seco suelto y Seco compacto.

Fig. 6. Preference of species for soil moisture. Saturated, Humid loose, Humid compact, Dry loose and Dry compact. 


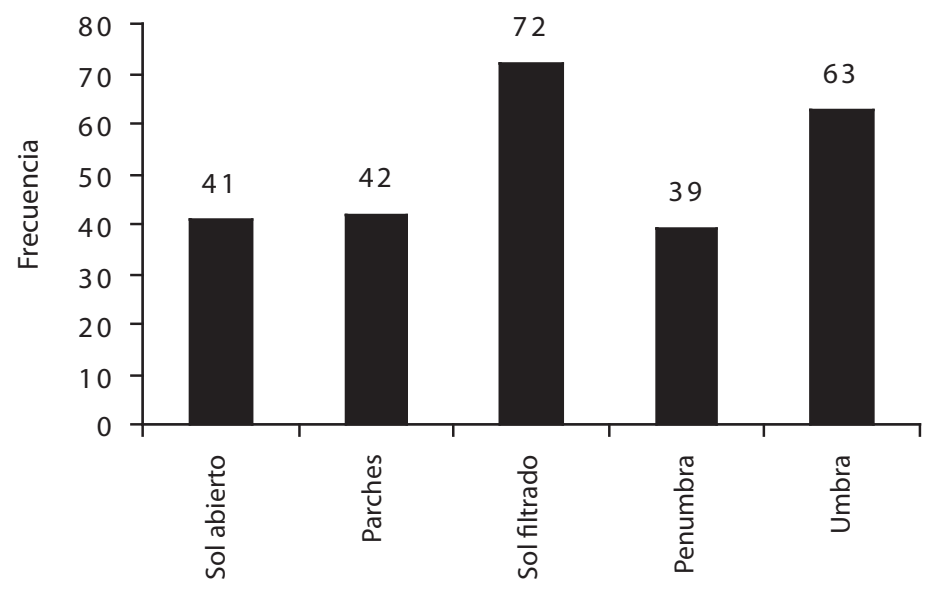

Iluminación

Fig. 7. Preferencia de las especies por las condiciones de iluminación. Sol abierto, Parches de sol, Sol filtrado, Penumbra y Umbra.

Fig. 7. Preference of communities for the conditions of illumination. Sunny, Patches of sun, Filtered sun, Partial shadow and Shadow.

épocas anteriores la diversidad pudo ser mayor, como es el caso de otras localidades estudiadas en la región Central del país, como la estación Biológica de Santa Maura, en el departamento de Jinotega, donde se han identificado unas 75 especies en un área de pocos kilómetros cuadrados (López 2003). Estos valores probablemente están influenciados por la existencia de un suelo mucho más rico en materia orgánica, así como un relieve más irregular que el existente de manera mayoritaria en la región del Pacífico.

Los valores de riqueza de especies aportados por este último autor son comparables con datos de localidades notables en diversidad malacológica en el nivel global, como los de Solem y Climo (1985), en la Península de Manukau, Nueva Zelanda, con 82 especies o los datos de Jaume (1944), en la zona de Viñales, provincia de Pinar del Río, Cuba, con más de 70 especies en un área más o menos similar a las de las localidades anteriormente citadas.

Existen datos sobre otras localidades de menor extensión pero también notables en cuanto a diversidad como los de Bidart et al.
(2003) para el Macizo montañoso de Topes de Collantes en la región central de Cuba, con 40 especies registradas; también los datos de Salas et al. (2003) sobre la malacofauna de los Yungas en el noroeste de la Argentina con 20 especies registradas, según estos autores la diversidad era notablemente más alta en esta región y ha disminuido considerablemente debido a la presión antropogénica existente.

Recientes resultados de Pérez et al. (2005, 2006) resultan también interesantes, ya que estos autores han reportado hasta el presente 56 especies de moluscos en fincas ganaderas de la región Centro-Norte del país, donde están monitoreando la diversidad de aves, plantas y moluscos; se debe señalar que esta región del país se encuentra mucho más conservada que la región del Pacífico.

Estos valores, en cambio, contrastan con los encontrados por Correa (1998), quien contabilizó 98 especies de gasterópodos terrestres en la región oriental de San Luis de Potosí, México. Aunque en todos los casos se debe tener en cuenta la disparidad de tamaño en las áreas estudiadas por los autores citados. 
En el presente trabajo se ha incluido también el análisis de un índice biogeográfico, ya que como mencionó Fontenla (1989) los estudios comunitarios actuales tienden a integrar cada vez más los enfoques ecológicos y zoogeográficos. El valor más alto del citado índice corresponde la cuadrícula 5 ( $\mathrm{IB}=3.04$ ) (Fig. 2), pero en la citada cuadrícula el valor de riqueza de especies no es el más alto del área de estudio. No obstante, en la cuadrícula 11 el IB=2.96, y en ella se presenta también el valor más alto de riqueza de especies $(\mathrm{S}=50)$ del área de estudio, en este sentido se debe enfatizar que, la utilización combinada del índice biogeográfico con los valores cuantitativos de la diversidad pueden constituir una herramienta muy poderosa para la estimación del valor conservacionista de las localidades de interés (Pérez y López 1995).

No obstante hay que reconocer que debido a la escala a que se realizó este estudio no se ha enfatizado en conocer la micro-distribución de los organismos, la cual es de gran ayuda en la comprensión de su papel ecológico e interacciones (Titmus 1983, Locasciulli y Boag 1987).

El endemismo encontrado en el área de estudio, de aproximadamente un $17 \%$, contradice los planteamientos que señalan a Nicaragua como un país de bajo endemismo, pero hay que tener en cuenta que esta afirmación se basa en análisis realizados sobre fauna vertebrada, aspecto que es frecuentemente explicado por el predominio de tierras bajas en el país, en las regiones del Pacífico y el Atlántico (Gillespie et al. 2001). Además, se debe tener en cuenta el alto grado de urbanización del área de estudio.

En otro ángulo de análisis hay un aspecto muy importante a tener en cuenta, y es que las especies de "amplia distribución" representan el $19 \%$ del conjunto de moluscos analizados, siendo, algunas de ellas, las de mayor abundancia registrada (Beckianum beckianum, Lamellaxis micra, Lamellaxis gracilis y Subulina octona). Consideramos que los conjuntos de especies transportadas por acción humana y nativas deben ser objeto de un estudio detallado, que pondría en evidencia el grado de sustitución de la fauna nativa por exótica y la degradación de los ambientes naturales.
En cuanto a la diversidad taxonómica de la región del Pacífico la familia más diversa es la familia Subulinidae, con 14 especies, seguida por las familias Spiraxidae (8 especies) y Vertiginidae (7 especies). Según datos preliminares de algunas localidades, la composición taxonómica de la región centro-norte del país es diferente a la encontrada en la región del Pacífico, y está marcada por el predominio de la familia Spiraxidae (López 2003).

Por otro lado, los valores de las abundancias obtenidas, con cinco especies muy abundantes (Subulina octona con 1021 indv; Bulimulus corneus, 931; Beckianum beckianum, 906; Lamellaxis gracilis, 893; Praticolella griseola, 828; y Lamellaxis micra; 494) y una gran parte de las especies con muy pocos individuos, sugiere un patrón de serie logarítmica, cuyo modelo predice un grupo pequeño de especies abundantes y una gran proporción de especies raras (Krebs 1978, Magurran 1987, Moreno 2000). Estos autores plantean que este modelo es aplicable cuando uno o unos pocos factores dominan la ecología de la comunidad como en las etapas iniciales de la sucesión en el caso de la vegetación.

Hábitat: según el análisis de la abundancia por formaciones vegetales, encontramos que existe una preferencia significativa por el bosque de galería $\left(\mathrm{x}^{2}=94.07, \mathrm{p}<0.05,10\right.$ $\mathrm{gl}$ ), asimismo el tipo de suelo, presenta una preferencia significativa por el suelo con hojarasca $\left(x^{2}=298.63, p<0.05,7 \mathrm{gl}\right)$. En este último aspecto nuestros resultados concuerdan con los de Getz y Uetz (1994), quienes encontraron una mayor diversidad de moluscos terrestres en los hábitat con mayor diversidad de hojarasca, no obstante en la opinión de estos autores el aumento de la diversidad se debe a la combinación de varios factores, entre los que ellos estudiaron humedad, elevación y diversidad de la hojarasca.

Según Uetz y Getz (1994) los valores extremos de humedad, mayor y menor, mostraron los valores más bajos de diversidad de especies, lo que también concuerda con nuestros resultados, en los que los valores de mayor 
diversidad estuvieron significativamente asociados con el suelo húmedo suelto $\left(x^{2}=36.94\right.$, $\mathrm{p}<0.01,4 \mathrm{gl})$ y mucho menos con el suelo húmedo compacto, habitualmente saturado. Los resultados del estudio cualitativo de la iluminación concuerdan con los obtenidos por Pérez et al. (1996), habiéndose obtenido una preferencia significativa por las condiciones sol filtrado $\left(x^{2}=17.66, p<0.05,4 \mathrm{gl}\right)$.

Un aspecto interesante a mencionar es que entre los "Asentamientos humanos", las especies mostraron una preferencia muy significativa por las Cercas vivas, en comparación con plantaciones de diferentes tipos por las que cabría esperar una mayor preferencia. Este aspecto fue retomado por Pérez (2002), para plantear que las Cercas vivas de diferentes tipos albergan una fauna notablemente diversa en sí misma y que, además, pueden funcionar como corredores manteniendo la conectividad entre las áreas protegidas y otros espacios naturales de la región.

Las comunidades de mayor diversidad en el Pacífico de Nicaragua se encuentran en las cuadrículas 3, 1113 y 16, a su vez, estas comunidades presentan un alto índice biogeográfico.

Estas comunidades con valores más altos de índice biogeográfico y de diversidad deben ser una prioridad diferencial al elaborar estrategias de conservación.

El hábitat idóneo para una comunidad de moluscos gasterópodos terrestres es aquél que presenta una iluminación de sol filtrado, vegetación de bosques de galería y suelo húmedo suelto con hojarasca. En el caso de ser comunidades asociadas con asentamientos humanos prefieren muy significativamente las cercas vivas.

\section{AGRADECIMIENTOS}

Los autores agradecemos a todas las personas que han estado involucradas de manera directa en los muestreos y el procesamiento de la información. A Kepa Altonaga (Universidad del País Vasco), por su apoyo en la parte taxonómica; a los estudiantes Zamira Guevara, Janina
Urcuyo, Johanna Carrillo, Cecilia Morales, Ana María Hernández, Freddy Ramírez y Bosco Bonilla. También agradecemos el extraordinario apoyo logístico de Rodrigo Ruiz y Marcos Rodríguez (Fac. Ciencia, Tecnología y Ambiente). Este proyecto no habría sido posible sin el apoyo de las autoridades de la Universidad Centroamericana, particularmente Mayra Luz Pérez y P. Xabier Gorostiaga SJ.

\section{RESUMEN}

Se estudiaron las comunidades de moluscos gasterópodos terrestres de la región del Pacífico de Nicaragua, con el método de cartografiado UTM. El área de estudio fue subdividida en 21 cuadrículas. Se recolectaron un total de 79 especies distribuidas en 43 géneros y 23 familias, lo que significa una alta riqueza de especies, géneros y familias. El valor más alto de riqueza de especies (S) correspondió a la cuadrícula 11 ( $\mathrm{S}=50$, Península de Chiltepe), y el valor más bajo a la cuadrícula 1 ( $\mathrm{S}=17$, Península de Cosigüina). El índice biogeográfico calculado con base en la información sobre distribución de las especies, presentó los valores más altos en las cuadrículas 5 (3.04), 6 (3.03), 8 (2.96) y 11(2.96). La cuadrícula 11 tuvo la mayor riqueza de especies $(\mathrm{S}=50)$ y uno de los mayores índices biogeográficos $(\mathrm{IB}=2.96)$. Esta cuadrícula contiene dos áreas protegidas y se encuentra en un estado de conservación relativamente bueno. El hábitat idóneo para una comunidad de moluscos gasterópodos terrestres presenta una iluminación de sol filtrado, asociado con bosques de galería y sustrato de suelo húmedo suelto con hojarasca.

Palabras clave: gasterópodos terrestres, Nicaragua, Vertiente Pacífica, diversidad, hábitat.

\section{REFERENCIAS}

Altonaga, K., B. Gómez, R. Martín, C. Prieto, A.I. Puente \& A. Rallo. 1994. Estudio faunístico y biogeográfico de los moluscos terrestres del norte de la península Ibérica. Eusko Legebiltzarra/ Parlamento Vasco, Vitoria, País Vasco, España.

Austin, M.P. 1985. Continuum concept, ordination methods, and niche theory. Ann. Rev. Ecol. Syst. 16: 39-61.

Bidart, L., W. Oliva y E. González. 2003. Malacofauna terrestre de Topes de Collantes, macizo montañoso Guamuahaya, Cuba central. Sociedad Brasilera de Malacología, Rio de Janeiro, Brasil. 
Brown, J.H. \& M.V. Lomolino. 1998. Biogeografía. Sinauer, Sunderland, Massachussetts, EEUU.

Calvo, M. 1994. Manual de preparación y conservación de invertebrados no artrópodos. Bouncopy S.A., Madrid, España.

Cameron, R.A.D. 1978. Life histories, density and biomass in a Woodland Snail community. J. Moll. Stud. 48: 159-166.

Cameron, R.A.D. 1982. Terrestrial snail faunas of the Malham area. Field studies 4: 715-728.

Cameron, R.A.D. 1986. Environment and diversities of forest snail faunas from coastal British Columbia. Malacologia 27: 341-355.

Correa Sandoval, A. 1999. Zoogeografía de los gastrópodos terrestres de la región oriental de San Luis Potosí, México. Rev. Biol. Trop. 47: 493-502.

Cowie, R.H., G.M. Nishida, Y. Basset \& S.M. GON III. 1995. Patterns of land snail distribution in a montane habitat on the island of Hawaii. Malacologia 36: 155-169.

Fischer, P. \& H. Crosse. 1870-1902. Mission scientifique au Mexique et dans L'Amerique Centrale. Mollusques Terrestres et Fluviatiles, Paris I, Francia.

Fontenla, J.L. 1989. Estructura taxonómica y zoogeografía de las mariposas (Rhopalocera) del Jardín Botánico de Cienfuegos, Cuba. Análisis comparativo. Poeyana 367: 1-24.

Getz, L.L. \& G.W. Uetz. 1994. Species diversity of terrestrial snails in the southern Appalachian mountains, U.S.A. Malacological Review 27: 61-74

Gillespie, T.W., K.E. Nicholson \& J. McCrary. 2001. Patterns of vertebrate species richness and conservation in Nicaragua. Natural Areas Journal 21: 159-167.

Incer, J. 1973. Geografía ilustrada de Nicaragua. Recalde, Managua, Nicaragua.

INEC. 1995. Censos Nacionales. Cifras oficiales finales. INEC, Managua, Nicaragua.

Jacobson, M.K. 1968. On a collection of terrestrial mollusks from Nicaragua. The Nautilus 81: 114-120.

Jaume, M. 1944. Excusión malacológica al Pan de Guajaibón I. Rev. Soc. Cub. Malacol. 3: 51-62.
Krebs, C.J. 1978. Ecología. Estudio de la distribución y la abundancia. Harla, México DF, México.

Locasciulli, O. \& D.A. Boag. 1987. Microdistribution of terrestrial snails (Stylommatophora) in forest litter. Can. Field-Nat. 101: 76-81.

López, A. 2003. Diversidad comparada de moluscos en diversas localidades de Nicaragua. Gaia 3: 1-9.

Ludwig, J.A. \& J.F. Reynolds. 1988. Statistical Ecology: a primer on methods and computing. A Wiley Interscience, Nueva York, EEUU.

Magurran, A.E. 1987. Ecological diversity and its measurement. Princeton University, Princeton, Nueva Jersey, EEUU.

MARENA. 1999. Reglamento de áreas protegidas de Nicaragua. Publicado en la Gaceta, Diario Oficial Nos. 42 y 43 del 2 y 3 de Marzo de 1999. MARENAPANIF, Managua, Nicaragua.

Marquet, R. 1985. An intensive zoogeographical and ecological survey of the land mollusca of Belgium: aims, methods and results (Mollusca: Gastropoda). Annls Soc. R. Zool. Belg. 115: 165-175.

Martens, EV. 1890-1901. Biologia Centrali-Americana. Land and Freshwater Mollusca. Taylor and Francis, Londres, Inglaterra.

Medina, A., C. Harvey, S. Vílchez, D. Sánchez \& B. Hernández. 2004. Diversidad y composición de Chiropteros en un paisaje fragmentado de bosque seco en Rivas, Nicaragua. Encuentro 68: 24-43.

Murgueitio, E., M. Ibrahim, E. Ramírez, A. Zapata, C.E. Mejía y F. Casasola. 2003. Usos de la tierra en fincas ganaderas. Apotema, Medellín, Colombia.

Moreno, C. 2000. Métodos para medir la biodiversidad. Manuales \& Tesis. Sociedad Entomológica de Aragón, Aragón, España.

Oviedo, E. 1993. Atlas Básico ilustrado de Nicaragua y el Mundo (ABINM). Epadisa-Salma, Madrid, España.

Pérez, A.M. 1999. Estudio Taxonómico y biogeográfico preliminar de la malacofauna continental (Mollusca: Gastropoda) del Pacífico de Nicaragua. Tesis Doctoral, Universidad del País Vasco/Euskal Herriko Unibertsitatea, País Vasco, España.

Pérez, A.M. 2002. Redes ecológicas: un uso alternativo del término y su aplicación a la conservación in situ. Un enfoque preliminar. Gaia 2: 1-10. 
Pérez, A.M. \& A. López. 1995. La diversidad malacológica en Nicaragua: aproximaciones a un nuevo enfoque. Encuentro 43: 59-72.

Pérez, A.M. \& A. López. 1999. Estudio taxonómico y biogeográfico preliminar de la malacofauna continental (Mollusca: Gastropoda) del Pacífico de Nicaragua (1995-1998). Cuadernos de Investigación, Universidad Centroamericana, No. 1: 1-52.

Pérez, A.M. \& A. López. 2002. Atlas de los moluscos continentales del Pacífico de Nicaragua. UCA, Managua, Nicaragua.

Pérez, A.M. \& A. López. 2003. Listado de la malacofauna continental del Pacífico de Nicaragua. Rev. Biol. Trop. 51: 405-461.

Pérez, A.M., J.C. Vilaseca \& N. Ziane. 1996. Sinecología básica de moluscos terrestres en cuatro formaciones vegetales de Cuba. Rev. Biol. Trop. 44: 133-146.

Pérez, A.M., M. Sotelo, F. Ramírez, I. Ramírez, A. López \& I. Siria. 2005. Composición y riqueza de aves, moluscos y plantas asociadas con sistemas silvopastoriles de Matiguás y Rio Blanco, Dpto de Matagalpa, Nicaragua. Encuentro 78: 136-165.

Pérez, A.M., M. Sotelo, F. Ramírez, A. López \& I. Siria. 2006. Conservación de la biodiversidad en sistemas silvopastoriles de Matiguás y Rio Blanco, Dpto de Matagalpa, Nicaragua. Ecosistemas 2006:1-16.

Salas, J.B. 1993. Arboles de Nicaragua. Hispamer, Managua, Nicaragua.

Salas, G., M.G. Cuezzo \& F. Romero. 2003. Biodiversidad de micromoluscos en selvas subtropicales de Tucumán, Argentina. Sociedad Brasilera de Malacología, Rio de Janeiro, Brasil.

Salgado, H. \& G. Páiz. 2004. Diversidad de la herpetofauna en la Reserva Natural Chocoyero-El Brujo, Ticuantepe, Managua. Encuentro 69:25-32.

Solem, A. \& F.M. Climo. 1985. Structure and habitat correlations of sympatric New Zealand land snail species. Malacologia 26: 1-30.

Titmus, G. 1983. Are animals populations really agregated. Oikos 40: 64-68.

Vílchez, S., C. Harvey, D. Sánchez, A. Medina \& B. Hernández. 2004. Diversidad de aves en un paisaje fragmentado. Encuentro 68: 60-75.

Walden, H. 1981. Communities and diversity of land mollusca in Scandinavian woodland. I. High diversities communities in taluses and boulder slopes in S.W. Sweden. J. Conchology 30: 351-377. 


\section{ANEXO 1}

Listado sistemático de las especies de moluscos terrestres presentes en el Pacífico de Nicaragua con sus categorías biogeográficas. Las abreviaturas corresponden a los nombres de las especies que posteriormente se reflejan en el Cuadro 2.

\section{CLASE GASTROPODA Cuvier, 1797}

FAMILIA Helicinidae Lamarck, 1799

Helicina rostrata Morelet, 1851 (Hr, Centroamericana)

Helicina tenuis (Pfeiffer, 1847) (Ht, Norteamericana y suramericana)

Lucidella lirata (Pfeiffer, 1847) (11, Norteamericana y suramericana)

FAMILIA Poteriidae Gray, 1850

Neocyclotus dysoni nicaraguense Bartsch \& Morrison, 1942 (Nd, Endémica)

FAMILIA Strobilopsidae Pilsbry, 1893

Strobilops sp. (Sp, Endémica)

FAMILIA Vertiginidae Fitzinger, 1833

Bothriopupa conoidea (Newcomb, 1853) (Bo, Norteamericana y suramericana)

Bothriopupa tenuidens (C. B. Adams, 1845) (Bte, Norteamericana y suramericana)

Pupisoma dioscoricola (C. B. Adams, 1845) (Pdi, Amplia distribución)

Pupisoma minus Pilsbry, 1920 (Pmi, Antillana)

Pupisoma mediomericana Autoridad y año (Pmed, Norteamericana y suramericana)

Sterkia antillensis Pilsbry, 1920 (San, Amplia distribución)

Vertigo milium (Gould, 1840) (Vmi, Norteamericana y suramericana)

FAMILIA Pupillidae Turton, 1831

Gastrocopta geminidens (Pilsbry, 1917) (Gge, Norteamericana y suramericana)

Gastrocopta gularis Thompson \& López, 1996 (Ggu, Endémica)

Gastrocopta servilis (Gould, 1843) (Gse, Antillana)

Gastrocopta pellucida (Pfeiffer, 1841) (Gpel, Antillana)

Gastrocopta pentodon (Say, 1821) (Gpen, Norteamericana y suramericana)

FAMILIA Succineidae Beck, 1837

Succinea guatemalensis Morelet, 1849 (Sgu, Centroamericana)

Succinea recisa (Morelet, 1851) (Sre, Centroamericana)

FAMILIA Ferussacidae Bourguignat, 1883

Cecilioides consobrinus Orbigny, 1855 (Cco, Centroamericana y Suramericana)

Cecilioides gundlachi (Pfeiffer, 1850) (Cgu, Antillana)

FAMILIA Subulinidae Crosse \& Fischer, 1877

Beckianum beckianum (Pfeiffer, 1846) (Bbe, Amplia distribución)

Beckianum sinistrum (Martens, 1898) (Bsi, Endémica)

Beckianum sp. (Bsp, Endémica)

Lamellaxis gracilis (Hutton, 1834) (Lgr, Amplia distribución)

Lamellaxis micra (Orbigny, 1835) (Lmi, Amplia distribución)

Leptinaria guatemalensis (Crosse \& Fischer, 1877) (Lgu, Centroamericana)

Leptinaria insignis ((Smith, 1898) (Li, Antillana)

Leptinaria interstriata (Tate, 1870) (Lint, Centroamericana)

Leptinaria lamellata (Potiez \& Michaud, 1838) (Lla, Antillana)

Leptinaria tamaulipensis Pilsbry, 1903 (Lta, Norteamericana y suramericana)

Leptinaria sp. (Lsp, Endémica)

Opeas pumilum (Pfeiffer, 1840) (Opu, Amplia distribución)

Pseudopeas sp. (Pssp, Endémica)

Subulina octona (Bruguière, 1792) (Soc, Amplia distribución) 
FAMILIA Streptaxidae Gray, 1860

Huttonella bicolor (Hutton, 1834) (Hbi, Amplia distribución)

FAMILIA Spiraxidae Baker, 1955

Euglandina cumingii (Beck, 1837) (Ecu, Centroamericana)

Euglandina obtusa (Pfeiffer, 1844) (Eob, Endémica)

Pittieria underwoodi (Fulton, 1897) (Pun, Centroamericana)

Salasiella guatemalensis Pilsbry, 1919 (Sgu, Centroamericana)

Salasiella hinkleyi Pilsbry, 1919 (Shi, Norteamericana y suramericana)

Salasiella perpusilla (Pfeiffer, 1880) (Spe, Norteamericana y suramericana)

Spiraxis sp. (Spsp, Endémica)

FAMILIA Limacidae Rafinesque, 1815

Deroceras laeve (Müller, 1774) (Dla, Amplia distribución)

FAMILIA Helicarionidae Bourguignat, 1888

Euconulus pittieri (Martens, 1892) (Epi, Centroamericana)

Guppya gundlachi (Pfeiffer, 1839) (Ggd, Amplia distribución)

Habroconus championi (Martens, 1892) (Hch, Centroamericana)

Habroconus selenkai (Pfeiffer, 1866) (Hse, Amplia distribución)

Habroconus trochulinus (Morelet, 1851) (Htr, Centroamericana)

FAMILIA Zonitidae Mörch, 1864

Glyphyalinia indentata (Say, 1822) (Gin, Norteamericana y suramericana)

Glyphyalinia sp. (Gsp, Endémica)

Hawaiia minuscula (Binney, 1840) (Hmi, Amplia distribución)

Striatura meridionalis (Pilsbry \& Ferriss, 1906), (Sme, Norteamericana y suramericana)

FAMILIA Helminthoglyptidae Pilsbry, 1939

Trichodiscina coactiliata (Deshayes, 1838) (Tco, Norteamericana y suramericana)

FAMILIA Polygyridae Pilsbry, 1895

Praticolella griseola (Pfeiffer, 1841) (Pg,r, Norteamericana y suramericana)

FAMILIA Thysanophoridae Pilsbry, 1926

Thysanophora hornii (Gabb, 1866) (Tho, Norteamericana y suramericana)

Thysanophora caecoides (Tate, 1870) (Tca, Centroamericana)

Thysanophora costaricensis Rehder, 1942 (Tcos, Centroamericana)

Thysanophora crinita (Fulton, 1917) (Tcr, Norteamericana y suramericana)

Thysanophora plagioptycha (Shuttleworth, 1854) (Tpl, Amplia distribución)

FAMILIA Sagdidae Pilsbry, 1895

Xenodiscula taintori (Goodrich \& Schalie, 1937, (Xta, Norteamericana y suramericana)

FAMILIA Bulimulidae Tryon, 1867

Bulimulus corneus (Sowerby, 1833) (Bco, Centroamericana)

Drymaeus alternans (Beck, 1837) (Dal, Centroamericana)

Drymaeus discrepans (Sowerby, 1833) (Ddi, Centroamericana)

Drymaeus dominicus Reeve, 1850 (Ddo, Amplia distribución)

Drymaeus multilineatus (Say, 1825) (Dmu, Norteamericana y suramericana)

Drymaeus translucens (Broderip, 1832) (Dtr, Centroamericana)

Drymaeus attenuatus (Pfeiffer,1853) (Datt, Norteamericana y suramericana)

FAMILIA Orthalicidae Pilsbry, 1899

Orthalicus ferussaci Martens, 1863 (Ofe, Norteamericana y suramericana)

Orthalicus princeps (Broderip, 1833) (Opr, Centroamericana) 
FAMILIA Systrophiidae Thiele, 1926

Drepanostomella pinchoti Pilsbry, 1930 (Dpi, Centroamericana)

Miradiscops opal (Pilsbry, 1916) (Mop, Endémica)

Miradiscops panamensis Pilsbry, 1930 (Mpa, Centroamericana)

FAMILIA Punctidae Morse, 1864

Punctum burringtoni Pilsbry, 1930 (Pbu, Centroamericana)

FAMILIA Charopidae Hutton, 1884

Chanomphalus pilsbryi (Baker, 1922) (Cpi, Norteamericana y suramericana)

Radiodiscus millecostatus Pilsbry \& Ferris, 1906 (Rmi, Norteamericana y suramericana)

Radiodiscus sp.,(Rsp, Endémica)

FAMILIA Veronicellidae Gray, 1840

Diplosolenodes occidentalis (Guilding, 1825) (Doc, Antillana)

Leidyula floridana (Leidy \& Binney, 1851) (Lfl, Amplia distribución) 